OPEN ACCESS

Edited by:

Marco Mirolli,

National Research Council (CNR), Italy

Reviewed by:

Luca Patanè

University of Catania, Italy

Malte Schilling,

Bielefeld University, Germany

*Correspondence:

Shura Suzuk

shura@riec.tohoku.ac.jp

Received: 17 September 2020 Accepted: 10 December 2020

Published: 08 January 2021

Citation:

Suzuki S, Kano T, ljspeert AJ and Ishiguro A (2021) Sprawling

Quadruped Robot Driven by

Decentralized Control With

Cross-Coupled Sensory Feedback

Between Legs and Trunk

Front. Neurorobot. 14:607455. doi: 10.3389/fnbot.2020.607455

\section{Sprawling Quadruped Robot Driven by Decentralized Control With Cross-Coupled Sensory Feedback Between Legs and Trunk}

\author{
Shura Suzuki ${ }^{1,2 *}$, Takeshi Kano ${ }^{1}$, Auke J. Ijspeert ${ }^{3}$ and Akio Ishiguro ${ }^{1}$ \\ ${ }^{1}$ Research Institute of Electrical Communication, Tohoku University, Sendai, Japan, ${ }^{2}$ Japan Society for the Promotion of \\ Science, Tokyo, Japan, ${ }^{3}$ Biorobotics Laboratory, École Polytechnique Fédérale de Lausanne, Lausanne, Switzerland
}

Quadruped animals achieve agile and highly adaptive locomotion owing to the coordination between their legs and other body parts, such as the trunk, head, and tail, that is, body-limb coordination. This study aims to understand the sensorimotor control underlying body-limb coordination. To this end, we adopted sprawling locomotion in vertebrate animals as a model behavior. This is a quadruped walking gait with lateral body bending used by many amphibians and lizards. Our previous simulation study demonstrated that cross-coupled sensory feedback between the legs and trunk helps to rapidly establish body-limb coordination and improve locomotion performance. This paper presented an experimental validation of the cross-coupled sensory feedback control using a newly developed quadruped robot. The results show similar tendencies to the simulation study. Sensory feedback provides rapid convergence to stable gait, robustness against leg failure, and morphological changes. Our study suggests that sensory feedback potentially plays an essential role in body-limb coordination and provides a robust, sensory-driven control principle for quadruped robots.

Keywords: sprawling locomotion, body-limb coordination, quadruped robot, decentralized control, sensory feedback control

\section{INTRODUCTION}

Quadrupeds freely locomote in their natural habitat with great agility and efficiency. This agility is achieved by coordination between their legs and other body parts such as the trunk, head, and tail, that is, by body-limb coordination (Hildebrand, 1959; Ashley-Ross, 1994; Reilly and Delancey, 1997; Ijspeert et al., 2007; Jagnandan and Higham, 2017; Ijspeert, 2020). However, most previous studies have intensively investigated interlimb coordination (Aoi et al., 2017), and less attention has been paid to the body-limb coordination mechanisms. A better understanding of these mechanisms can contribute to the design of agile quadruped robots and help to interpret the motor control of quadruped animals.

When investigating body-limb coordination mechanisms, sprawling locomotion can be seen to be a remarkable behavior. A sprawling walking gait combines lateral bending of the body with leg movements. This is exhibited by many amphibians and lizards (Hildebrand, 1959; AshleyRoss, 1994; Reilly and Delancey, 1997; Ijspeert et al., 2007; Jagnandan and Higham, 2017; Ijspeert, 2020). Lateral bending during locomotion provides a longer stride and stronger thrust, and this 
behavior was implemented by the first terrestrial quadrupeds (Nyakatura et al., 2019; Ijspeert, 2020). Therefore, this locomotion mode is likely to contain an important characteristic of the body-limb coordination mechanisms.

Sprawling locomotion in vertebrate animals is controlled by a distributed neural network called the central pattern generator (CPG) and sensory feedback from peripheral nerves, according to experiments with salamanders (Cabelguen et al., 2003). Based on these findings, several neural network models have been proposed for sprawling robots to emulate and investigate sprawling locomotion (Ijspeert et al., 2007; Harischandra et al., 2011; Crespi et al., 2013; Yin et al., 2016; Zhong et al., 2018). However, most of the models were based on open-loop control. Thus, the extent to which sensory feedback contributes to shaping body-limb coordination was not investigated, particularly in studies using real robots. Clarifying the role of sensory feedback in sprawling locomotion is an important step in understanding the fundamental principles of body-limb coordination.

Our motivation is to understand the role of sensory feedback in sprawling locomotion. Our previous study proposed decentralized control with cross-coupled sensory feedback, that is, bidirectional feedback from body to limb and limb to body in a simulated robot (Suzuki et al., 2019). The simulated results showed that sensory feedback helps to rapidly establish appropriate body-limb coordination. Moreover, sensory feedback provides adaptability to leg failure and changes in the body aspect ratio. This paper presents the experimental validation of the proposed control using a developed quadruped robot. The robot was designed based on the simulated robot in the previous study, and it was equipped with servo motors with built-in torque sensors. The results show a tendency similar to that of the simulation. This suggests that cross-coupled sensory feedback potentially plays an essential role in bodylimb coordination. It could be a useful concept for designing decentralized and robust controllers for quadruped robots.

The remainder of this paper is structured as follows. Section 2 describes the developed quadruped robot and decentralized control with cross-coupled sensory feedback. Section 3 describes the experimental setup, results, and discussion. In section 4 , the conclusions and recommendations for future studies are presented.

\section{ROBOT AND CONTROL ALGORITHM}

\subsection{Mechanical System}

Figure 1A shows the developed quadruped robot Twister with nine actuated degrees of freedom (DoFs): one actuated DoF in the trunk and two actuated DoFs per leg. It is primarily constructed using 3D-printed ABS (acrylonitrile-butadiene-styrene) resin pieces. The actuated DoFs were realized with servomotors (Dynamixel XM430-W350-R, ROBOTIS, stall torque 4.1 [N.m] at $12 \mathrm{~V}$ ) that were controlled by a single-board computer (Raspberry Pi 4 Model B, 4 GB RAM, OKdo). The robot consists of a trunk segment and four leg segments. The trunk segment has a rotary actuator in the yaw direction. Each leg segment has two rotary actuators in the yaw and roll directions, along with a phase oscillator that controls the leg segment.

Current and angle sensors are included in each servomotor. The current values are proportional to the motor torque, so the current sensor is used as a torque sensor. An angle sensor at the trunk detects the trunk-joint angle $\theta^{b} . \theta^{b}$ is positive when the trunk-joint bends to the right, as shown in Figure 1B.

\subsection{Control Algorithm}

This section describes decentralized control with cross-coupled sensory feedback slightly modified from the control algorithm in our previous study (Suzuki et al., 2019), for robot control. The controller is made from oscillators, which represent CPGs. Unlike most CPG controllers, the controller does not use interoscillator couplings but sensory-couplings through bidirectional feedback between the legs and the trunk (Figure 2A). The sensory couplings were achieved using the following three feedback rules:

1. Torque sensory feedback from limb to limb

2. Torque sensory feedback from body to limb

3. Torque sensory feedback from limb to body

The first rule is responsible for coordinating the four legs as they move forward while supporting the body. The second and third rules comprise bidirectional feedback that establishes selforganized body-limb coordination. The controller generates stable and flexible sprawling locomotion by the combination of oscillators generating rhythmic motion and the feedback rules coordinating the movements of bodily DoFs. While the controller is programmed here on a single computer, it is ideally suited for a distributed implementation on different independent microcontrollers, for example, one for the trunk and one per leg, with minimal communication between microcontrollers sharing sensory signals. The following section describes the control algorithm and the effects of sensory feedback.

\subsubsection{Leg Control}

A phase oscillator is implemented in each leg, and its phase determines the target angle of the rotary actuators in the yaw and roll directions as follows:

$$
\begin{aligned}
\bar{\theta}_{i}^{y} & =C_{0}^{y}-C_{a m p}^{y} \cos \phi_{i}, \\
\bar{\theta}_{i}^{r} & =C_{0}^{r}-C_{a m p}^{r} \sin \phi_{i},
\end{aligned}
$$

where $\bar{\theta}_{i}^{y}$ and $\bar{\theta}_{i}^{r}$ denote the target angles, $C_{0}^{y}$ and $C_{0}^{r}$ represent the neutral angles, $C_{a m p}^{y}$ and $C_{a m p}^{r}$ represent the amplitude of the yaw and roll actuators, respectively (Figure 1B). $\phi_{i}$ is the oscillator phase. When $0<\phi_{i}<\pi$, the leg is in the swing phase; otherwise, it is in the stance phase. The suffix $i$ denotes the leg identifier (1: left fore, 2: right fore, 3: left hind, and 4: right hind). The time 
A

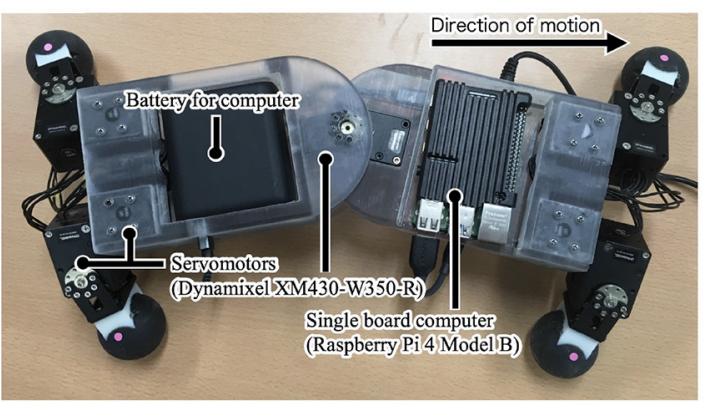

B

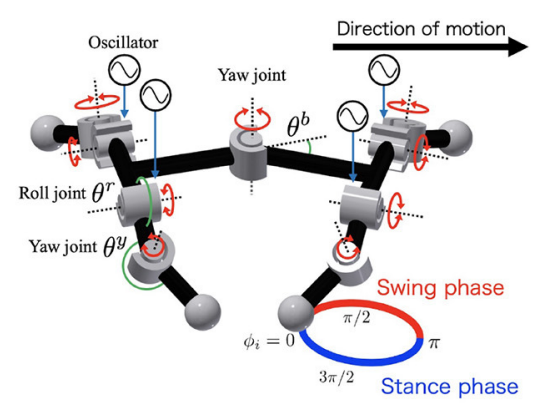

FIGURE 1 | (A) Twister, a quadruped robot that exploits lateral bending. Body height $0.09 \mathrm{~m}$, width $0.095 \mathrm{~m}$, length $0.30 \mathrm{~m}$, leg length $0.07 \mathrm{~m}$, and weight $2.1 \mathrm{~kg}$. (B) Schematic of the robot. The trunk has one servomotor, and each leg has two servomotors controlled by the phase of the oscillators $\phi_{i} . \theta^{b}$ is the trunk angle, and $\theta^{y}$ and $\theta^{r}$ are the angles of the leg actuators in the yaw and roll directions, respectively.

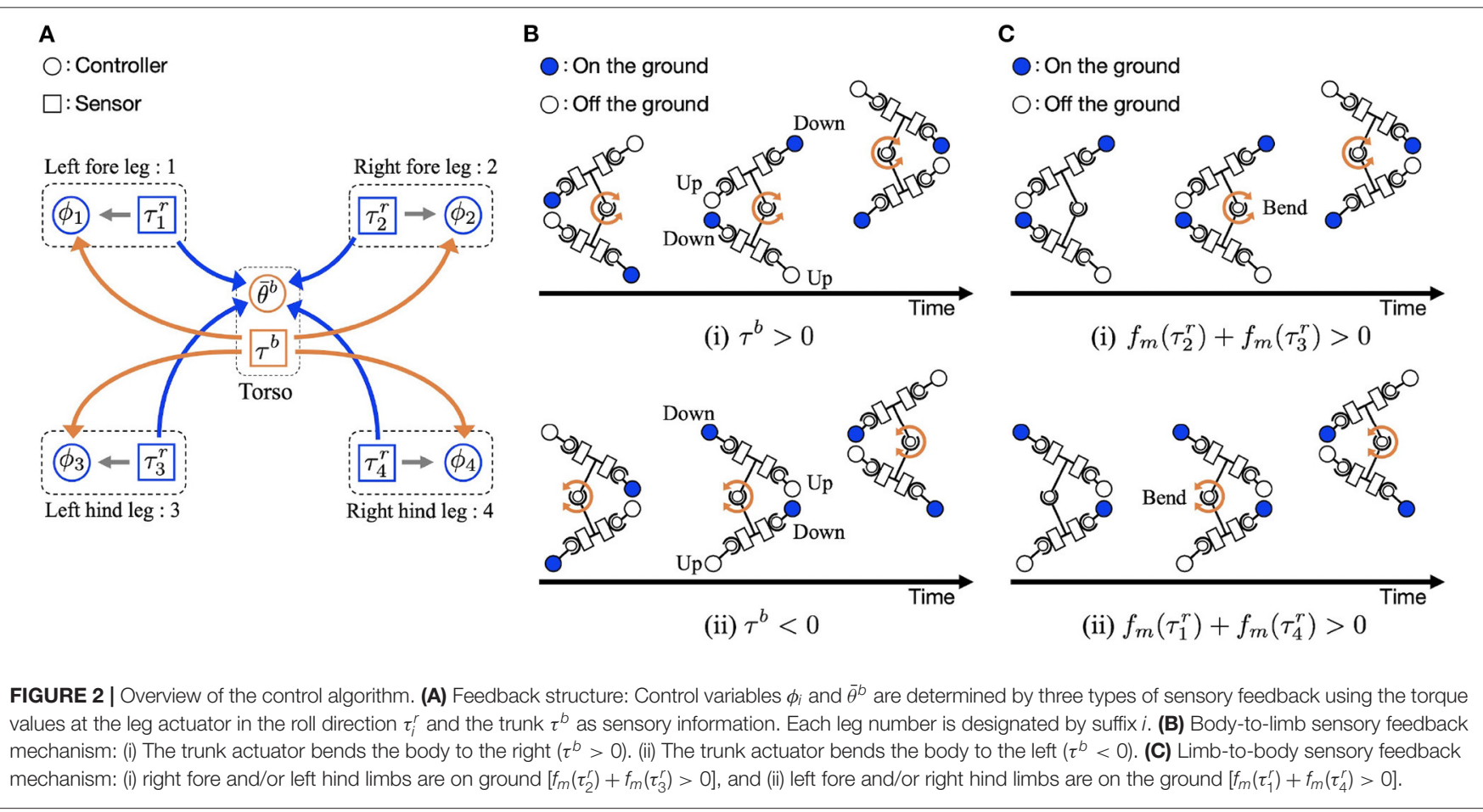

evolution of the phase is described as follows:

$$
\begin{aligned}
\dot{\phi}_{i} & =\omega+f_{L L, i}+f_{B L, i}, \\
f_{L L, i} & =-\sigma_{L L} f_{m}\left(\tau_{i}^{r}\right), \\
f_{B L, i} & = \begin{cases}\sigma_{B L} \tau^{b} \cos \phi_{i} & (i=1,4) \\
-\sigma_{B L} \tau^{b} \cos \phi_{i} & (i=2,3),\end{cases} \\
f_{m}\left(\tau_{i}^{r}\right) & =\max \left[0, \tau_{i}^{r}-\tau_{t h}^{r}\right],
\end{aligned}
$$

where $\omega[\mathrm{rad} / \mathrm{s}]$ denotes the intrinsic angular velocity of the phase oscillators, and $\sigma_{L L}[\mathrm{rad} / \mathrm{N} \cdot \mathrm{m} \cdot \mathrm{s}]$ and $\sigma_{B L}[\mathrm{rad} / \mathrm{N} \cdot \mathrm{m} \cdot \mathrm{s}]$ are the weights of the sensory feedback terms. $\tau_{i}^{r}[\mathrm{~N} \cdot \mathrm{m}]$ and
$\tau^{b}[\mathrm{~N} \cdot \mathrm{m}]$ represent the torques at the leg actuator in the roll direction and at the trunk actuator, respectively. $f_{m}\left(\tau_{i}^{r}\right)$ is correlated with the extent to which the leg supports the body. Thus, $f_{m}\left(\tau_{i}^{r}\right)$ substitutes for the ground reaction force (GRF) information. $\tau_{t h}^{r}[\mathrm{~N} \cdot \mathrm{m}]$ is a constant positive value as the threshold of the sensors.

Equation (3) works according to the feedback from limb to limb. The local feedback rule was proposed by Owaki et al. (2013). It generates adaptive interlimb coordination in response to the speed and physical properties of the robot (Owaki et al., 2013; Owaki and Ishiguro, 2017). Based on the sensory feedback effect, the oscillator phase is modulated to $3 \pi / 2$ when $f_{m}\left(\tau_{i}^{r}\right)>0$. When the leg supports the body, the roll joint of that limb has higher torque signals, that is, higher $f_{m}\left(\tau_{i}^{r}\right)$. Thus, this feedback 
means that the leg remains on the ground when it supports the body. The local sensory information $f_{m}\left(\tau_{i}^{r}\right)$ describes the extent to which a specific leg provides support to the body, and also indicates how much other legs are currently contributing to supporting the body. Using the sensory information, this feedback can generate adaptive interlimb coordination without neural communication between the legs.

Equation (4) relates to the feedback from the body to the limb (Figure 2B). When the trunk actuator bends the body to the right $\left(\tau^{b}>0\right)$, the oscillator phases of the left fore and right hind legs are modulated toward $\pi / 2$ to lift the legs, and the oscillator phases of the right fore and left hind legs are modulated toward $3 \pi / 2$ to place the legs on the ground. By phase modification, the left fore and right hind legs lift from the ground, and the other legs are anchored on the ground. This facilitates the trunk actuator bending the body to the right $\left(\theta^{b}>0\right)$, and the robot moves forward when the anchored legs serve as a pivot.

\subsubsection{Body Control}

The time evolution of the target angle of the trunk actuator is described as follows:

$$
\begin{aligned}
\dot{\bar{\theta}}^{b} & =a\left(-\theta^{b}+f_{L B, i}\right), \\
f_{L B, i} & =\sigma_{L B} \tanh \left\{\rho\left(-f_{m}\left(\tau_{1}^{r}\right)+f_{m}\left(\tau_{2}^{r}\right)+f_{m}\left(\tau_{3}^{r}\right)-f_{m}\left(\tau_{4}^{r}\right)\right)\right\},
\end{aligned}
$$

where $a[1 / \mathrm{s}]$ represents the reciprocal of the time constant. Variables $\theta^{b}$ and $\bar{\theta}^{b}$ are the actual angle and target angle of the trunk actuator, respectively. $\sigma_{L B}[\mathrm{rad}]$ and $\rho[1 / \mathrm{N} \cdot \mathrm{m}]$ represent the weights of the sensory feedback.

Equation (7) relates to the feedback from limb to body. The sensory feedback effect is that the trunk bends in response to ground contact, as shown in Figure 2C. When the right fore and/or left hind limbs are on the ground $\left[f_{m}\left(\tau_{2}^{r}\right)+f_{m}\left(\tau_{3}^{r}\right)>0\right]$, the actuator makes the right side of the body concave $\left(\tau^{b}>0\right.$, Figure 2Ci). Similarly, when the left fore and/or right hind limbs are on the ground $\left[f_{m}\left(\tau_{1}^{r}\right)+f_{m}\left(\tau_{4}^{r}\right)>0\right]$, the trunk actuator makes the left side of the body concave ( $\tau^{b}<0$, Figure 2Cii). The interaction of the sensory feedback from body to limb and limb to body establishes the relationship between the legs and trunk, providing longer strides and more powerful pushing off against the ground.

\section{ROBOT EXPERIMENTS}

To verify the control algorithm in the real world, we conducted three experiments: (i) steady locomotion, (ii) fault tolerance, and (iii) robustness to change in body aspect ratio. All experiments were conducted on flat terrain and recorded with a video camera (Cyber-shot DSC-RX0M2, Sony). The video of representative results can be referred to in Supplementary Material. The parameter values were as follows: $C_{0}^{y}=\pi$ [rad], $C_{0}^{r}=7 \pi / 6$ $[\mathrm{rad}], C_{a m p}^{y}=\pi / 12[\mathrm{rad}], C_{a m p}^{r}=\pi / 12$ [rad], $\omega=1.5 \pi$ $[\mathrm{rad} / \mathrm{s}], \sigma_{L L}=6.83[\mathrm{rad} / \mathrm{N} \cdot \mathrm{m} \cdot \mathrm{s}], \sigma_{B L}=2.28[\mathrm{rad} / \mathrm{N} \cdot \mathrm{m} \cdot \mathrm{s}]$, $\sigma_{L B}=\pi / 6[\mathrm{rad}], \rho=0.05[1 / \mathrm{N} \cdot \mathrm{m}], \tau_{t h}^{r}=0.088[\mathrm{~N} \cdot \mathrm{m}]$, $a=5.0[1 / \mathrm{s}]$. Here, most of the parameter values were set to be identical to those in our simulation study (Suzuki et al., 2019). However, several parameters were adjusted from these values by trial-and-error because of differences in the mechanical and morphological properties between the real and simulated robots. The analysis was conducted using MathWorks' MATLAB for gait classification and Tracker, a free video analysis and modeling tool, for speed derivation.

\subsection{Steady Locomotion}

First, we observed the manner of locomotion from two points of view: footfall patterns and the angle of the trunk-joint. The results are shown in Figure $\mathbf{3 A}$ and Supplementary Video 1. Figure $3 \mathbf{A}$ show the time evolution of (top) the trunk-joint angle $\theta^{b}$ and (bottom) the gait diagrams, where the colored region represents the stance phases $\left[f_{m}\left(\tau_{i}^{r}\right)>0\right]$. The duty factor is $61.0 \%$, and the diagonality is $42.2 \%$. The duty factor is the time percentage that one foot spends in the stance phase during a gait cycle, and diagonality is the percentage of the cycle period by which the left/right hind footfall precedes the left/right fore footfall. Thus, the resulting gait is classified as a lateral-sequence walking gait, in which the feet touch down in the order right hind $(\mathrm{RH})$, right fore (RF), left hind (LH), and left fore (LF), in Hildebrand's gait classification (Hildebrand, 1965; Cartmill et al., 2002). It is qualitatively similar to the sprawling locomotion shown in animals (Ashley-Ross, 1994; Reilly and Delancey, 1997). In addition, the robot converged to this gait in a few steps, even though we set the initial phase of all oscillators to be the same $\left(\phi_{1}=\phi_{2}=\phi_{3}=\phi_{4}=3 \pi / 2\right.$, Supplementary Video 1$)$. This is due to the effect of the feedback. (Owaki et al., 2013) showed that the feedback from limb to limb (Equation 3) provides smoothly interlimb coordination. Additionally, in our model, the feedback from body to limb and from limb to body (Equation 4, Figure 2B) helps establish stable locomotion with body-limb coordination. This means that the robot produces the appropriate relationship between the legs and trunk spontaneously and immediately without a predefined body-limb relationship, as in conventional studies. This was the same as in the simulation results (Suzuki et al., 2019).

Next, we investigated the contribution of the cross-coupled sensory feedback to locomotion performance. We derived the locomotion speed and CoT from the tracking data and compared them for the proposed control with and without cross-coupled sensory feedback between the legs and trunk $\left(\sigma_{L B}=\sigma_{B L}=\right.$ $0)$. Here, steady locomotion without cross-coupled sensory feedback is shown in Figure 3B and Supplementary Video 2. Stable walking was also exhibited in a few steps, because the controller still includes the limb to limb sensory feedback. The gait is classified as a walking trot gait, the duty factor is $53.2 \%$, and the diagonality is $48.5 \%$. The CoT is calculated as follows:

$$
\operatorname{CoT}=\frac{1}{D m g} \int_{0}^{T} \sum P_{i}(t) \mathrm{d} t,
$$

where $D[\mathrm{~m}]$ is the travel distance, $m[\mathrm{~kg}]$ is the total mass of the robot, $g\left[\mathrm{~m} / \mathrm{s}^{2}\right]$ is the gravitational acceleration, and $P_{i}(t)[\mathrm{W}]$ is the power consumption of each servomotor. Figures 3C,D show the mean speed and CoT for each control mechanism, where the 
A

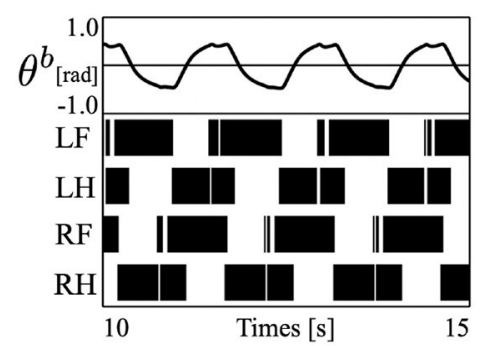

B

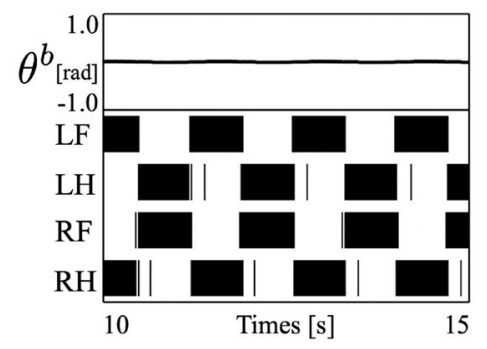

C

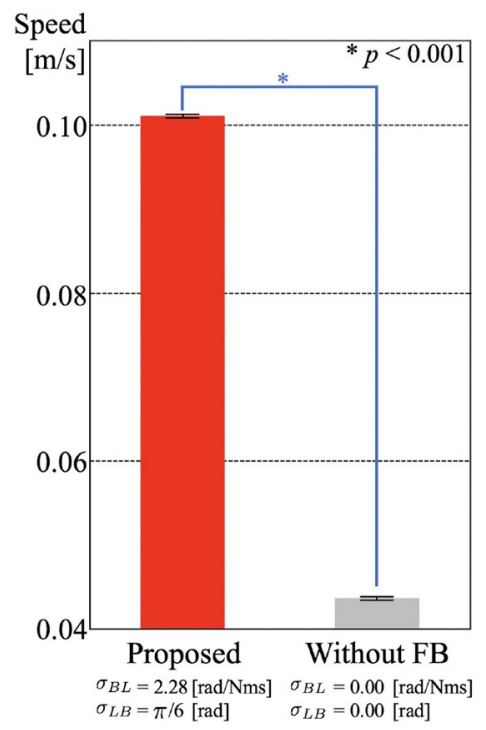

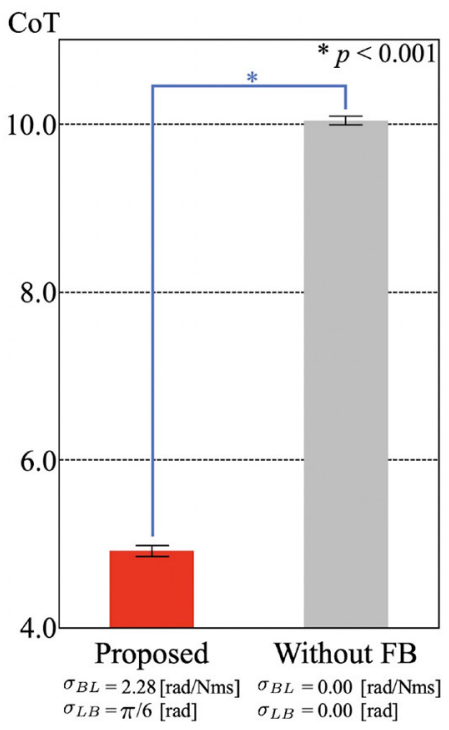

FIGURE 3 | Experimental results of section 3.1. (A) Gait pattern of proposed control. Time evolution of (top) trunk-joint angle $\theta^{b}$ and (bottom) gait diagrams. The colored region in the gait diagrams represents the stance phase of each leg $\left[f_{m}\left(\tau_{i}^{r}\right)>0\right]$. (B) Gait pattern of the control without cross-coupled sensory feedback $\left[\sigma_{L B}=\sigma_{B L}=0\right]$. (C) Locomotion speed of the proposed control $(0.1010[\mathrm{~m} / \mathrm{s}], \mathrm{SD}=0.0002)$, and without cross-coupled sensory feedback $(0.0436 \mathrm{~m} / \mathrm{s}, \mathrm{SD}=$ 0.0002). The bar heights and the error bars indicate means and standard deviations (SD) for five trials, respectively $\left({ }^{*} p<0.001, t\right.$-test). (D) Cost of transport (CoT) of the proposed control (CoT $=4.91, \mathrm{SD}=0.0646)$, and without cross-coupled sensory feedback $(\mathrm{CoT}=10.04, \mathrm{SD}=0.0487)$.

height of the plots and the error bars represent the mean values and standard deviations (SD) for five trials, respectively. We used Welch's $t$-test as a statistical test to compare the controllers. From the perspectives of locomotion speed and CoT, the proposed control mechanism achieved significantly higher speeds and energy efficiency than those of the control mechanism without cross-coupled sensory feedback $(p<0.001)$. The results show that lateral bending of the body with leg movements improves mobility, and that cross-coupled sensory feedback contributes to this.

\subsection{Fault Tolerance}

In this experiment, we investigated the robustness to leg failure. Here, leg failure means that the leg becomes stuck in a particular position. Specifically, the phase of the leg oscillator is fixed to $3 \pi / 2$, and therefore, the target angle of the leg actuator is also fixed to $\bar{\theta}_{i}^{y}=C_{0}^{y}, \bar{\theta}_{i}^{r}=C_{0}^{r}+C_{a m p}^{r}$, respectively. We derived the locomotion speed and CoT for the proposed control mechanism under the foreleg $\left(\phi_{1}=\phi_{2}=3 \pi / 2\right)$ and hindleg failure conditions, $\left(\phi_{3}=\phi_{4}=3 \pi / 2\right)$. These were compared with those of the open-loop trot control mechanism to consider the importance of sensory feedback in an unexpected situation.

The open-loop trot control mechanism predefined the relationship between the legs and the trunk without any sensory feedback. Each actuated DoF has a phase oscillator that defines the target angle as follows:

$$
\begin{aligned}
\dot{\phi}_{i} & =\omega^{\prime}, \\
\phi_{b} & =\phi_{1}, \\
\bar{\theta}^{b} & =C_{a m p}^{b} \sin \phi_{b},
\end{aligned}
$$

where $\omega^{\prime}[\mathrm{rad} / \mathrm{s}]$ denotes the intrinsic angular velocity of the phase oscillators. $\phi_{b}$ [rad] is the phase of the oscillator in the trunk, and $C_{a m p}^{b}$ represents the amplitude of the trunk actuator. The parameters $\omega^{\prime}$ and $C_{a m p}^{b}$ were defined to refer to the gait cycle and the trunk-joint angle of the proposed model (Figure 3A), as follows: $\omega=4.363[\mathrm{rad} / \mathrm{s}]$ and $C_{a m p}^{b}=0.43[\mathrm{rad}]$. The initial conditions of the oscillator are $\phi_{1}=\phi_{4}=0, \phi_{2}=\phi_{3}=\pi$. Steady locomotion with the open-loop control mechanism is shown in Supplementary Video 3.

The locomotion during each leg failure condition is shown in Supplementary Videos 4-7. Figure 4 shows the locomotion speed and CoT of the proposed and open-loop trot control under intact, foreleg failure, and hindleg failure conditions. The proposed control showed significantly higher speeds and energy efficiency than the open-loop trot control under the leg failure conditions $(p<0.001)$, although the opposite tendency was observed in the intact condition. This result suggests that the sensory feedback mechanism works well to adapt to unexpected bodily damage, although it does not yield the same performance as the open-loop control when intact.

\subsection{Robustness to Changes in Body Aspect Ratio}

We observed locomotion when the leg length was elongated (length $=0.14[\mathrm{~m}]$ ) to investigate the robustness to variations in the body aspect ratio. The observed locomotion is shown in Supplementary Video 8. The mean speed was $1.735[\mathrm{~m} / \mathrm{s}]$, and CoT was 5.25 for five trials. The robot with long legs showed stable locomotion similar to the one with short legs (length $=0.07$ 


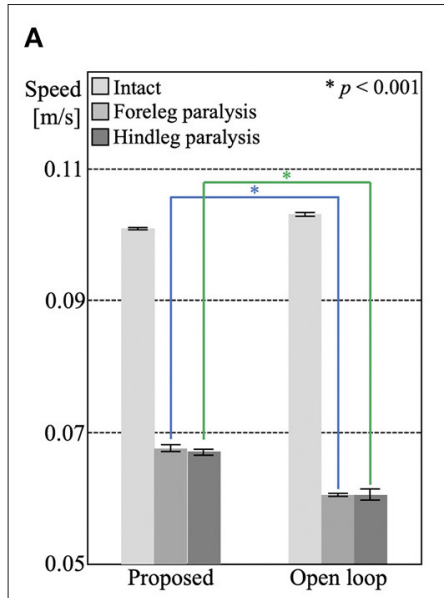

\section{B}

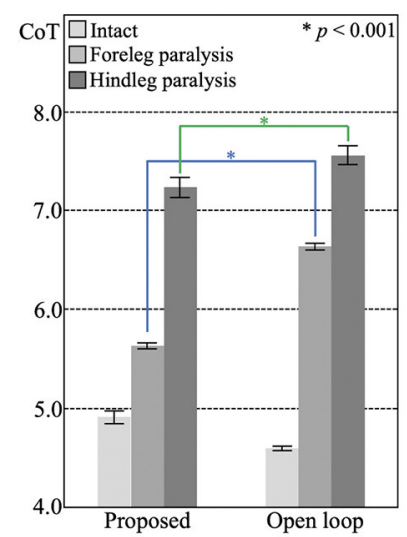

FIGURE 4 | The experimental results of section 3.2. The bar heights and the error bars indicate the means and SD for five trials, respectively ${ }^{*} p<0.001$, $t$-test). (A) Locomotion speed of the proposed control under the intact condition $(0.1010[\mathrm{~m} / \mathrm{s}], \mathrm{SD}=0.0002)$, foreleg failure condition $(0.0675[\mathrm{~m} / \mathrm{s}]$, $\mathrm{SD}=0.0005)$, and hindleg failure condition $(0.0670[\mathrm{~m} / \mathrm{s}], \mathrm{SD}=0.0004)$, and of open-loop trot control under the intact condition $(0.1031 \mathrm{~m} / \mathrm{s}, \mathrm{SD}=$ $0.0003)$, foreleg failure condition $(0.0605 \mathrm{~m} / \mathrm{s}, \mathrm{SD}=0.0002)$, and hindleg failure condition $(0.0605[\mathrm{~m} / \mathrm{s}], \mathrm{SD}=0.0008)$. (B) CoT of the proposed control under the intact condition (COT $=4.91, \mathrm{SD}=0.0646$ ), foreleg failure condition $(\mathrm{CoT}=5.63, \mathrm{SD}=0.031)$, and hindleg failure condition $(\mathrm{COT}=7.24, \mathrm{SD}=$ $0.102)$, and of open-loop trot control under intact condition (CoT $=4.59, \mathrm{SD}=$ 0.0211 ), foreleg failure condition ( $\mathrm{CoT}=6.63, \mathrm{SD}=0.036$ ), and hindleg failure condition (CoT $=7.56, \mathrm{SD}=0.095)$.

[m], Supplementary Video 1). Moreover, it did not require any changes in the control parameters for the morphological changes. This highlights the robustness of the control algorithm against variations in the body aspect ratios.

\section{CONCLUSION AND FUTURE WORK}

We have developed a quadruped robot and demonstrated via experiments that decentralized control with cross-coupled sensory feedback (Suzuki et al., 2019) enables effective sprawling locomotion. Unlike most previous works based on CPGs with inter-oscillator couplings (Ijspeert et al., 2007; Crespi et al., 2013; Yin et al., 2016; Ijspeert, 2020), or on gait patterns based on geometric mechanics (Zhong et al., 2018), our model uses sensory-couplings through bidirectional feedback between the legs and trunk (Figure 2A). Owing to this mechanism, the robot can quickly converge to stable gaits, achieve high locomotion performances, and adapt to leg failure. Interestingly, these gaits are obtained through an emergent property of controllerbody-environment interactions unlike the fixed gait patterns of previous work. And the generated gaits are highly similar to those found to be optimal in terms of forward speed through geometric mechanics (Zhong et al., 2018). Our results suggest that the sensory feedback mechanisms at the peripheral level play an important role in coordinating the body and limbs.

This work will also provide insight into motor control underlying other legged locomotion. Many modeling studies of six-legged walking have investigated the importance of sensory feedback (Manoonpong et al., 2013; Schilling et al., 2013; Dürr et al., 2019; Schilling and Cruse, 2020). In particular, Schilling et al. (2013) proposed a decentralized mechanism similar to that used in this study. This indicates the similarities of motor control between six-legged and four-legged locomotion. Through examining the importance of body motion in legged locomotion, Schilling et al. (2012) indicated the significance of trunk-joints in six-legged walking and turning, and Ly et al. (2011) showed that the bio-inspired vertebral column enhances humanoid balance. Furthermore, Fukuhara et al. and Kano et al. used similar feedback mechanisms, and found that the body bending along the pitching direction increases locomotion speeds of a cheetah-like robot (Fukuhara et al., 2020) and sea roach robot (Kano et al., 2019), respectively. Our results also suggest the importance of sensory feedback and body-limb coordination in legged locomotion. This indicates that further study of crosscoupled sensory feedback would contribute to understanding legged locomotion not limited to sprawling locomotion.

This work also contributes to the field of robotics. Most previous studies on legged robots mainly focused on leg motion rather than whole-body motion. In contrast, this study achieved effective locomotion with lateral bending using a simple control framework. Furthermore, because the framework was designed for decentralized implementation, it can provide fault tolerance and robustness with low computational cost and local sensing. Therefore, this work is expected to provide fundamental information for the next paradigm of fault-tolerant legged robots.

Finally, we point out several problems and limitations with this work and potential solutions for them. First, a neurophysiological basis for the proposed model is still lacking. Although it is known that the neural circuit for limb movements is located in the particular vertebrae above and below the axial trunk network (Bicanski et al., 2013; Ryczko et al., 2020), it is still unclear whether salamanders share proprioceptive information between the legs and trunk for locomotion, as in the proposed controller. This needs to be further investigated. Second, the parameters have not been explored sufficiently as compared with our simulation study (Suzuki et al., 2019). As a consequence, it is still unclear to what extent the behavior is sensitive to parameter changes. Clarifying this will help identify the crucial parameters for locomotion. Third, the experiments were limited to straight walking, and turning behaviors were not investigated. We expect that the turning direction can be controlled by modulating the feedback from limbs to body in an asymmetric way (for example, the robot would turn right by removing the sensory information for the left foreleg). Fourth, the robot developed in this study has only one DoF in the trunk, whereas salamanders have many DoFs. In the future, we would like to implement control in a robot with a multi-DoF trunk and compare its behavior with that of real salamanders.

\section{DATA AVAILABILITY STATEMENT}

The original contributions presented in the study are included in the article/Supplementary Material, further inquiries can be directed to the corresponding author/s. 


\section{AUTHOR CONTRIBUTIONS}

SS, AJI, and AI designed the study. AI supervised the project. SS developed the robot platform. SS, TK, and AI designed the control algorithm. SS carried out the robot experiments and the initial draft of the manuscript. All authors revised the manuscript.

\section{FUNDING}

This work was supported by the Human Frontier Science Program (RGP0027/2017), Japan Science and Technology Agency, CREST (JP-MJCR14D5), the Grant-in-Aid for JSPS Research Fellows (No. 20J10805), and the Division

\section{REFERENCES}

Aoi, S., Manoonpong, P., Ambe, Y., Matsuno, F., and Wörgötter, F. (2017). Adaptive control strategies for interlimb coordination in legged robots: a review. Front. Neurorobot. 11:39. doi: 10.3389/fnbot.2017. 00039

Ashley-Ross, M. (1994). Hindlimb kinematics during terrestrial locomotion in a salamander (dicamptodon tenebrosus). J. Exp. Biol. 193, 255-283.

Bicanski, A., Ryczko, D., Knuesel, J., Harischandra, N., Charrier, V., Ekeberg, Ö., et al. (2013). Decoding the mechanisms of gait generation in salamanders by combining neurobiology, modeling and robotics. Biol. Cybern. 107, 545-564. doi: 10.1007/s00422-012-0543-1

Cabelguen, J.-M., Bourcier-Lucas, C., and Dubuc, R. (2003). Bimodal locomotion elicited by electrical stimulation of the midbrain in the salamander notophthalmus viridescens. J. Neurosci. 23, 2434-2439. doi: 10.1523/JNEUROSCI.23-06-02434.2003

Cartmill, M., Lemelin, P., and Schmitt, D. (2002). Support polygons and symmetrical gaits in mammals. Zool. J. Linn. Soc. 136, 401-420. doi: 10.1046/j.1096-3642.2002.00038.x

Crespi, A., Karakasiliotis, K., Guignard, A., and Ijspeert, A. J. (2013). Salamandra robotica II: An amphibious robot to study salamander-like swimming and walking gaits. IEEE Trans. Robot. 29, 308-320. doi: 10.1109/TRO.2012.223 4311

Dürr, V., Arena, P. P., Cruse, H., Dallmann, C. J., Drimus, A., Hoinville, T., Krause, T., et al. (2019). Integrative biomimetics of autonomous hexapedal locomotion. Front. Neurorobot. 13:88. doi: 10.3389/fnbot.2019. 00088

Fukuhara, A., Koizumi, Y., Suzuki, S., Kano, T., and Ishiguro, A. (2020). Decentralized control mechanism for body-limb coordination in quadruped running. Adapt. Behav. 28, 151-164. doi: 10.1177/10597123198 65180

Harischandra, N., Knuesel, J., Kozlov, A., Bicanski, A., Cabelguen, J.-M., Ijspeert, A., et al. (2011). Sensory feedback plays a significant role in generating walking gait and in gait transition in salamanders: a simulation study. Front. Neurorobot. 5:3. doi: 10.3389/fnbot.2011.0 0003

Hildebrand, M. (1959). Motions of the running cheetah and horse. J. Mammal. 40, 481-495. doi: 10.2307/1376265

Hildebrand, M. (1965). Symmetrical gaits of horses. Science 150, 701-708. doi: $10.1126 /$ science.150.3697.701

Ijspeert, A. J. (2020). Amphibious and sprawling locomotion: from biology to robotics and back. Annu. Rev. Control Robot. Auton. Syst. 3, 173-193. doi: 10.1146/annurev-control-091919-0 95731

Ijspeert, A. J., Crespi, A., Ryczko, D., and Cabelguen, J.-M. (2007). From swimming to walking with a salamander robot driven by a spinal cord model. Science 315, 1416-1420. doi: 10.1126/science.1138353 for Interdisciplinary Advanced Research and Education, Tohoku University.

\section{ACKNOWLEDGMENTS}

The authors would like to thank Dr. Akira Fukuhara, Kotaro Yasui, Taishi Mikami, and Hayato Amaike of Tohoku University for their helpful suggestions.

\section{SUPPLEMENTARY MATERIAL}

The Supplementary Material for this article can be found online at: https://www.frontiersin.org/articles/10.3389/fnbot. 2020.607455/full\#supplementary-material

Jagnandan, K., and Higham, T. E. (2017). Lateral movements of a massive tail influence gecko locomotion: an integrative study comparing tail restriction and autotomy. Sci. Rep. 7, 1-8. doi: 10.1038/s41598-01711484-7

Kano, T., Ikeshita, Y., Fukuhara, A., and Ishiguro, A. (2019). Bodylimb coordination mechanism underlying speed-dependent gait transitions in sea roaches. Sci. Rep. 9, 1-9. doi: 10.1038/s41598-019-39 $862-3$

Ly, O., Lapeyre, M., and Oudeyer, P.-Y. (2011). "Bio-inspired vertebral column, compliance and semi-passive dynamics in a lightweight humanoid robot," in 2011 IEEE/RSJ International Conference on Intelligent Robots and Systems (San Francisco, CA), 1465-1472. doi: 10.1109/IROS.2011.60 95019

Manoonpong, P., Parlitz, U., and Wörgötter, F. (2013). Neural control and adaptive neural forward models for insect-like, energy-efficient, and adaptable locomotion of walking machines. Front. Neural Circ. 7:12. doi: 10.3389/fncir.2013.00012

Nyakatura, J. A., Melo, K., Horvat, T., Karakasiliotis, K., Allen, V. R., Andikfar, A., et al. (2019). Reverse-engineering the locomotion of a stem amniote. Nature 565, 351-355. doi: 10.1038/s41586-018-0851-2

Owaki, D., and Ishiguro, A. (2017). A quadruped robot exhibiting spontaneous gait transitions from walking to trotting to galloping. Sci. Rep. 7, 1-10. doi: 10.1038/s41598-017-00348-9

Owaki, D., Kano, T., Nagasawa, K., Tero, A., and Ishiguro, A. (2013). Simple robot suggests physical interlimb communication is essential for quadruped walking. J. R. Soc. Interface 10:20120669. doi: 10.1098/rsif.2012. 0669

Reilly, S., and Delancey, M. (1997). Sprawling locomotion in the lizard sceloporus clarkii: the effects of speed on gait, hindlimb kinematics, and axial bending during walking. J. Zool. 243, 417-433. doi: 10.1111/j.1469-7998.1997. tb02791.x

Ryczko, D., Simon, A., and Ijspeert, A. J. (2020). Walking with salamanders: From molecules to biorobotics. Trends Neurosci 43, 916-930. doi: $10.1016 /$ j.tins.2020.08.006

Schilling, M., and Cruse, H. (2020). Decentralized control of insect walking: a simple neural network explains a wide range of behavioral and neurophysiological results. PLoS Comput. Biol. 16:e1007804. doi: 10.1371/journal.pcbi.1007804

Schilling, M., Hoinville, T., Schmitz, J., and Cruse, H. (2013). Walknet, a bio-inspired controller for hexapod walking. Biol. Cybern. 107, 397-419. doi: 10.1007/s00422-013-0563-5

Schilling, M., Paskarbeit, J., Schmitz, J., Schneider, A., and Cruse, H. (2012). "Grounding an internal body model of a hexapod walker control of curve walking in a biologically inspired robot," in 2012 IEEE/RSJ International Conference on Intelligent Robots and Systems (Vilamoura), 2762-2768. doi: 10.1109/IROS.2012.638 5709 
Suzuki, S., Kano, T., Ijspeert, A. J., and Ishiguro, A. (2019). Decentralized control with cross-coupled sensory feedback between body and limbs in sprawling locomotion. Bioinspir. Biomimet. 14:066010. doi: 10.1088/1748-3190/ ab3ef6

Yin, X.-Y., Jia, L.-C., Wang, C., and Xie, G.-M. (2016). The effect of waist twisting on walking speed of an amphibious salamander like robot. Acta Mech. Sin. 32, 546-550. doi: 10.1007/s10409-015-0532-4

Zhong, B., Aydin, Y. O., Gong, C., Sartoretti, G., Wu, Y., Rieser, J. M., et al. (2018). "Coordination of back bending and leg movements for quadrupedal locomotion," in Robotics: Science and Systems (Pittsburgh, PA). doi: 10.15607/RSS.2018.XIV.020
Conflict of Interest: The authors declare that the research was conducted in the absence of any commercial or financial relationships that could be construed as a potential conflict of interest.

Copyright $($ () 2021 Suzuki, Kano, Ijspeert and Ishiguro. This is an open-access article distributed under the terms of the Creative Commons Attribution License (CC BY). The use, distribution or reproduction in other forums is permitted, provided the original author(s) and the copyright owner(s) are credited and that the original publication in this journal is cited, in accordance with accepted academic practice. No use, distribution or reproduction is permitted which does not comply with these terms. 